04

\title{
Особенности формирования многополосной функции пропускания на базе многочастотной акустооптической дифракции
}

\author{
() В.В. Проклов ${ }^{1}$, Ю.Г. Резвов 2 Ф \\ ${ }^{1}$ Фрязинский филиал Института радиотехники и электроники им. В.А. Котельникова РАН, \\ 141190 Фрязино, Россия \\ ${ }^{2}$ Новомосковский институт (филиал), \\ Российский химико-технологический университет им. Д.И. Менделеева, \\ 301665 Новомосковск, Россия \\ ฯ e-mail: rezvovyug@mail.ru \\ Поступила в редакцию 08.08.2017 г.
}

\begin{abstract}
Получено аналитическое решение для функции пропускания некогерентного широкополосного излучения при акустооптической (AО) фильтрации с использованием дискретного множества монохроматических $\mathrm{AO}$ волн с малым перекрытием их спектров. Исследованы характеристики АО преобразования сплошного спектра некогерентного излучения в заданное множество дискретных узких полос спектрального пропускания при возбуждении дискретного набора звуковых частот. Проведен анализ функций пропускания отдельных каналов с учетом частичного перекрытия их спектральных линий и возможных при этом интермодуляционных частотных искажений. Показано, что при фотоэлектрическом преобразовании и детектировании дифрагированного света на электронном выходе образуется стационарное значение среднеквадратичной мощности приходящего потока света. Установлено, что на этой основе на выходе АО фильтра можно сформировать необходимую стационарную многополосную, почти эквидистантную, функцию пропускания устройства путем формирования соответствующего спектра акустического возбуждения. Выяснены специфические особенности данного метода формирования многополосной функции пропускания: ограничение максимально достижимой эффективности дифракции в отдельно взятом канале, возможность ослабления связи боковых лепестков соседних каналов и др. Проведено моделирование многополосного акустооптического фильтра на основе монокристалла парателлурита $\left(\mathrm{TeO}_{2}\right)$, с которым ранее проводились эксперименты по оптическому спектральному кодированию информации. При этом достигнуто удовлетворительное согласие теоретических и экспериментальных данных.
\end{abstract}

DOI: $10.21883 /$ OS.2018.01.45367.178-17

\section{Введение}

В последние годы наблюдается большой интерес к исследованиям многочастотной акустооптической (AO) дифракции, в результате которых были установлены как новые эффекты таких взаимодействий, так и наметились новые пути их использования [1-5]. Так, например, в области многоканальной $\mathrm{AO}$ модуляции лазерного излучения в ряде работ были найдены причины возникновения вредных интермодуляционных искажений при многочастотном взаимодействии [6], а также и возможности существенного их подавления на базе подобающего выбора геометрии анизотропной АО дифракции в кристаллической среде и соответствующего синтеза управляющих акустических сигналов [7,8]. Возникли предложения по использованию многочастотной $\mathrm{AO}$ дифракции для программируемой фильтрации широкополосных потоков некогерентного излучения, способной выполнять определенные виды адаптивной спектральной обработки оптических сигналов, включая увеличение контраста изображения объектов в присутствии известных типов фоновых помех, коррекцию формы коротких оптических импульсов и т.п. [9-11].

Следует отметить также недавно появившиеся данные о перспективных возможностях по использованию многочастотного АО взаимодействия в разработках новых систем оптических телекоммуникаций на принципе передачи данных с кодовым разделением каналов (O-CDMA - code division multiple access), а также использование многополосной АО фильтрации излучения в системах удаленной идентификации объектов по принципу многополосной фильтрации спектрально сложных оптических сигналов $[12,13]$.

Вместе с тем в настоящее время большой интерес к более широкому применению новых методов обработки информации на базе многополосного акустооптического фильтра (МАОФ) ограничивается в своем развитии отсутствием развитой теории, описывающей основные характеристики таких устройств и условия их реализации, что и явилось поводом для постановки и проведения данной работы.

\section{1. О стационарной функции пропускания при акустооптической фильтрации света в поле монохроматического звука}

В рассматриваемой модели акустическое поле представляется в виде пучка с параллельными границами 

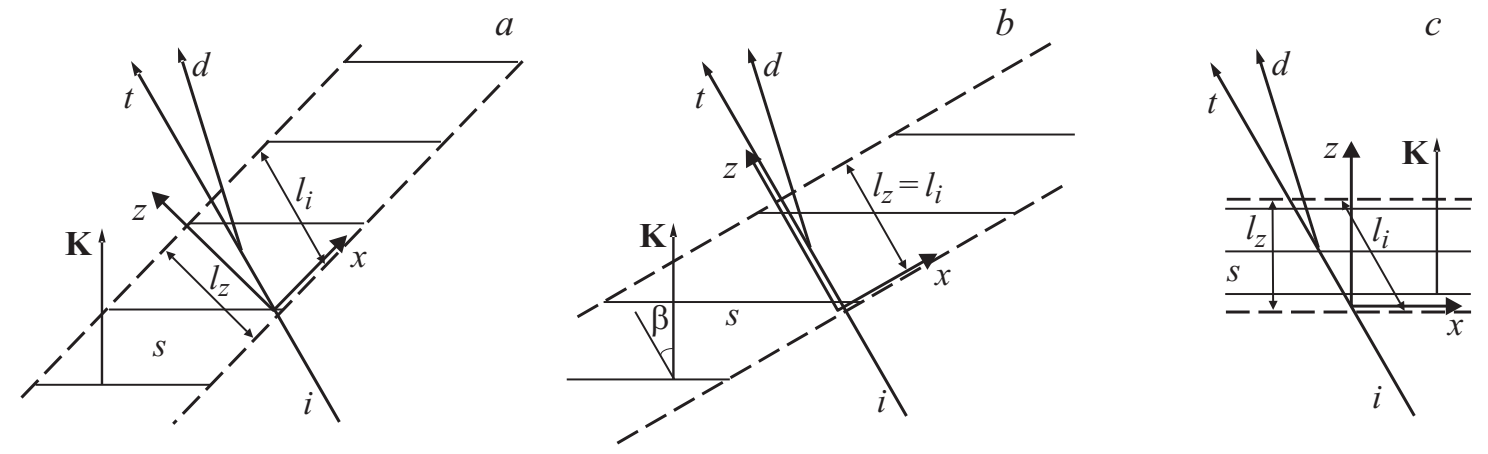

Рис. 1. Эквивалентные геометрии АО взаимодействия в анизотропной среде.

(штриховые линии на рис. $1, a-c$ ). Падающий линейно поляризованный световой луч (далее отмечаемый индексом „і“) пересекает акустический пучок (,s“). В результате анизотропной брэгговской дифракции световой луч расщепляется на проходящий, с исходной поляризацией $\left(, t^{\text {“ }}\right)$, и дифрагированный $\left(, d^{\text {“ }}\right)$, который поляризован ортогонально падающему и распространяется под небольшим углом к проходящему.

Световые лучи могут распространяться вдоль направления распространения звука (коллинеарная и квазиколлинеарная геометрии АО взаимодействия) или пересекать его под значительными углами. Поскольку известно, что амплитуды плоских волн, составляющих световые лучи, меняются в направлении нормали к границе акустического возмущения [14-18], ось $z$ целесообразно направить вдоль этой нормали, ось $x$ - вдоль границы акустического столба в плоскости АО дифракции $x z$, а ось $y$ - перпендикулярно этой плоскости.

Можно показать, что в случае пересечения столба под произвольным углом результат АО дифракции почти эквивалентен случаю пересечения другого акустического столба (это подтверждается анализом работ $[19,20])$. Этот эквивалентный столб должен иметь те же параметры звука, за исключением ширины столба и ориентации параллельных границ. Ширина столба должна меняться синхронно с ориентацией границ таким образом, чтобы оставалось постоянным расстояние $l_{i}$ вдоль пересекаемого падающим световым пучком акустического возмущения. Исключением из такой возможности эквивалентной трансформации является случай почти касательного пересечения светом исходного или эквивалентного столба. Таким образом, мы можем выбрать наиболее удобную форму столба в каждом конкретном случае. В частности, исходная задача (рис. 1,a) может быть рассмотрена в случае ортогонального падения света на эквивалентный возмущенный слой (рис. $1, b)$ и в случае ориентации границ столба параллельно фронту звуковой волны (рис. 1, $c$ ).

Конкретно будем рассматривать задачу в условиях, соответствующих рис. $1, b$. В данном случае центральный волновой вектор $\mathbf{k}_{i}$ падающего светового пучка направлен по оси $z$, длина взаимодействия $l_{z}$ совпадает с расстоянием $l_{i}$. Угол $\beta$ между волновым вектором звука $\mathbf{K}$ и осью $z$ может принимать значения в широких пределах. В частности, этот угол равен нулю при коллинеарной дифракции, а при квазиколлинеарной дифракции совпадает с углом сноса звука.

Акустическое поле представляет собой монохроматическую бегущую волну, так что поле смещений в области взаимодействия есть реальная часть выражения $A \exp \left\{j\left(K_{x} x+K_{z} z-\Omega t\right)\right\}$, где $A-$ комплексная амплитуда, $\Omega$ - циклическая частота звука, $K_{x}=\frac{\Omega}{v_{s}} \sin \beta$, $K_{z}=\frac{\Omega}{v_{s}} \cos \beta, v_{s}-$ скорость звука.

Пусть на акустический пучок в плоскости АО дифракции $x z$ падает монохроматический световой луч, поперечное распределение амплитуды в котором (при $z=0)$ имеет вид $E_{i}(x, y)$. Если циклическая частота падающего света $\omega_{t}$, то проекции волнового вектора центральной волны равны соответственно $k_{t z}=k_{t}=\frac{2 \pi n_{t}}{\lambda}$, $k_{t x}=k_{t y}=0$, где $\lambda-$ длина волны в вакууме, $n_{t}-$ показатель преломления. Будем считать (аналогично плосковолновой модели), что центральная плоская волна дифрагированного светового пучка определяется параметрами $\omega_{d}=\omega_{t}+\Omega, k_{d x}=K_{x}, k_{d y}=0, k_{d z}=\sqrt{k_{d}^{2}-k_{d x}^{2}}$, $k_{d}=\frac{2 \pi n_{d}}{\lambda}$. Возможное нарушение АО синхронизма описывает выражение $\Delta k_{z}=k_{t z}-k_{d z}-K_{z}$. Таким образом, оптическое поле в среде представлено совокупностью проходящего и дифрагированного световых пучков:

$$
\begin{aligned}
\mathbf{E}(x, y, z, t)= & \sum_{p=t, d} B_{p} \mathbf{e}_{p} E_{p}(x, y, z) \\
& \times \exp \left\{j\left(k_{p x} x+k_{p z} z-\omega_{p} t\right)\right\} .
\end{aligned}
$$

Здесь $\mathbf{e}_{t, d}-$ единичные векторы поляризации, $E_{t, d}(x, y, z)$ - медленно меняющиеся амплитуды пучков. Поток энергии каждого пучка распространяется с групповой скоростью $\mathbf{S}_{t, d}$ (рассчитанной для центральной волны), который вследствие анизотропии среды может незначительно отклоняться от волнового вектора. Кроме того, при анизотропной дифракции скорости $S_{t, d}$ могут заметно различаться. В (1) для удобства введен множитель $B_{d}=\left(\left(\mathbf{S}_{d}, \mathbf{k}_{d}\right) S_{d z} /\left(\mathbf{S}_{t}, \mathbf{k}_{t}\right) S_{t z}\right)^{1 / 2}$, учитывающий разную скорость распространения энергии в среде, при 
этом $B_{t}=1$. При таком подходе эффективность дифракции в произвольной плоскости $z=$ const определяется формулой

$$
\eta=\frac{\int\left|E_{d}(x, y, z)\right|^{2} d x d y}{\int\left|E_{i}(x, y, z)\right|^{2} d x d y}
$$

Амплитуды $E_{t, d}(x, y, z)$ меняются при распространении как вследствие АО взаимодействия, так и дифракционных эффектов, вызванных ограниченностью апертуры. Чтобы выделить эффект, обусловленный первой причиной, перейдем к пространственно-спектральному представлению. Полагаем, что каждый пучок образован набором плоских волн, амплитуды которых зависят (в результате АО взаимодействия) от координаты $z$. Тогда пространственный спектр оптического поля в плоскости $z=$ const (2-мерное фурье-преобразование выражения (1) по переменным $x$ и $y$ ) имеет вид

$$
\begin{aligned}
\mathbf{E}\left(k_{x}, k_{y}, z, t\right)= & \sum_{p=t, d} B_{p} \mathbf{e}_{p} U_{p}\left(k_{p x}^{\prime}, k_{p y}^{\prime}, z\right) \\
& \times \exp \left\{j\left(k_{p z}+k_{p z}^{\prime}\right) z-j \omega_{p} t\right\} .
\end{aligned}
$$

Здесь $U_{p}\left(k_{x}, k_{y}, z\right)$ - зависящий от $z$ и отнесенный к опорной плоскости $z=0$ спектр амплитуды $E_{p}(x, y, z)$, $k_{p x}^{\prime}=k_{x}-k_{p x}$ - отклонение проекции волнового вектора от центрального значения, аналогичен смысл величин $k_{p y}^{\prime}, k_{p z}^{\prime}$.

В отсутствие звука спектры $U_{p}$ не зависят от $z$, а зависимость $k_{p z}^{\prime}\left(k_{p x}^{\prime}, k_{p y}^{\prime}\right)$ определяет эволюцию амплитуды $E_{p}(x, y, z)$. В линейном приближении эта зависимость определяет снос пучка, в параболическом описывает также дифракционные искажения. С учетом малости оптической анизотропии и углов падения-дифракции приближенно можно написать

$$
k_{p z}^{\prime}=-\left(S_{p x} / S_{p z}\right) k_{p x}^{\prime}-\left(k_{p x}^{\prime 2}+k_{p y}^{\prime 2}\right) / 2 k_{p} .
$$

Вследствие фотоупругого эффекта световые лучи в акустическом поле связаны между собой, причем количественной мерой этого эффекта (в случае точного АО синхронизма) являются коэффициенты связи $q_{t, d}$, пропорциональные амплитуде звука, а также зависящие от геометрии взаимодействия, параметров среды и характеристик взаимодействующих волн. С учетом введенного в (1), (2) множителя $B_{d}$ различие коэффициентов между собой исчезает, т.е. $q_{t}=q_{d}=q$. Далее, будем считать коэффициент АО связи комплексным, фаза которого равна фазе комплексной звуковой амплитуде. С учетом выражений (1)-(3) и сделанных замечаний стандартная процедура дает уравнения эволюции спектров взаимодействующих оптических пучков:

$$
\begin{gathered}
\frac{d U_{d}\left(k_{x}^{\prime}, k_{y}^{\prime}, z\right)}{d z}=\frac{q}{2} U_{t}\left(k_{x}^{\prime}, k_{y}^{\prime}, z\right) \exp (2 j \varphi z), \\
\frac{d U_{t}\left(k_{x}^{\prime}, k_{y}^{\prime}, z\right)}{d z}=-\frac{q^{*}}{2} U_{d}\left(k_{x}^{\prime}, k_{y}^{\prime}, z\right) \exp (-2 j \varphi z),
\end{gathered}
$$

где

$$
2 \varphi=\Delta k_{z}+\left(\frac{S_{d x}}{S_{d z}}-\frac{S_{t x}}{S_{t z}}\right) k_{x}^{\prime}+\frac{\left(k_{x}^{\prime 2}+k_{y}^{\prime 2}\right)}{2 k_{d}}-\frac{\left(k_{x}^{\prime 2}+k_{y}^{\prime 2}\right)}{2 k_{t}} .
$$

Последнее выражение можно упростить, учитывая, что с точностью до знака параметр $\varphi_{1}=\frac{S_{d x}}{S_{d z}}-\frac{S_{t x}}{S_{t z}}$ равен малому углу между групповыми скоростями световых пучков. Отметим также, что разность обратных волновых чисел можно написать в виде

$$
\frac{1}{k_{d}}-\frac{1}{k_{t}}=\frac{\lambda}{2 \pi}\left(\frac{1}{n_{d}}-\frac{1}{n_{t}}\right)=\frac{\lambda}{2 \pi} \Delta\left(n^{-1}\right),
$$

где $\Delta\left(n^{-1}\right)=\frac{1}{n_{d}}-\frac{1}{n_{t}}$. Таким образом, используя два малых параметра $\varphi_{1}$ и $\Delta\left(n^{-1}\right)$, имеем

$$
\varphi=\frac{1}{2} \Delta k_{z}+\frac{\varphi_{1}}{2} k_{x}^{\prime}+\frac{\lambda \Delta\left(n^{-1}\right)}{8 \pi}\left(k_{x}^{\prime 2}+k_{y}^{\prime 2}\right) .
$$

Так как система (4) является линейной относительно неизвестных спектров, удобно ввести спектральные характеристики $T_{t, d}$, определяемые соотношением $U_{t, d}\left(k_{x}^{\prime}, k_{y}^{\prime}, z\right)=T_{t, d}\left(k_{x}^{\prime}, k_{y}^{\prime}, z\right) U_{i}\left(k_{x}^{\prime}, k_{y}^{\prime}\right)$, где $U_{i}\left(k_{x}^{\prime}, k_{y}^{\prime}\right) \propto \int E_{i}(x, y) \exp \left(-j k_{x}^{\prime} x-j k_{y}^{\prime} y\right) d x d y-$ пространственный спектр поперечного распределения амплитуды падающего пучка света. Граничные условия для полученной системы имеют вид: $T_{t}\left(k_{x}^{\prime}, k_{y}^{\prime}, 0\right)=1$, $T_{d}\left(k_{x}^{\prime}, k_{y}^{\prime}, 0\right)=0$.

Введем ряд безразмерных переменных: длина взаимодействия $Z=\frac{z}{l_{z}}$ (меняется в пределах $\left.0 \leq Z \leq 1\right)$, $V=\frac{q l_{z}}{2}-$ комплексная связь, $\delta=\frac{1}{2} \Delta k_{z} l_{z}$ - расстройка для центральных волн световых пучков,

$$
F=\varphi l_{z}=\delta+\frac{\varphi_{1}}{2} l_{z} k_{x}^{\prime}+\frac{\lambda l_{z} \Delta\left(n^{-1}\right)}{8 \pi}\left(k_{x}^{\prime 2}+k_{y}^{\prime 2}\right) .
$$

Теперь система (4) с учетом (5) примет вид

$$
\frac{d T_{d}}{d Z}=V T_{t} \exp (2 j F Z), \quad \frac{d T_{t}}{d Z}=-V^{*} T_{d} \exp (-2 j F Z) .
$$

Она имеет аналитическое решение:

$$
\begin{gathered}
T_{d}=\frac{V}{\sqrt{|V|^{2}+F^{2}}} \sin \left(\sqrt{|V|^{2}+F^{2}} Z\right) \exp (j F Z), \\
T_{t}=\left(\cos \left(\sqrt{|V|^{2}+F^{2}} Z\right)+\frac{j F}{\sqrt{|V|^{2}+F^{2}}}\right. \\
\left.\quad \times \sin \left(\sqrt{|V|^{2}+F^{2}} Z\right) \exp (-j F Z)\right) .
\end{gathered}
$$

Можно отметить, что система (6) и решение (7) для спектров пучков написаны в виде, максимально близком к хорошо изученному случаю плосковолнового приближения. Таким образом, формулы (7) с учетом исходных ограничений дают точное аналитическое выражение пространственно-спектральных характеристик $T_{t, d}\left(k_{x}^{\prime}, k_{y}^{\prime}, Z\right)$ в однородном акустическом поле. Это позволяет рассчитать эффективность дифракции (с учетом теоремы Парсеваля) в виде

$$
\eta=\frac{\int\left|U_{i}\left(k_{x}^{\prime}, k_{y}^{\prime}\right)\right|^{2}\left|T_{d}\left(k_{x}^{\prime}, k_{y}^{\prime}, 1\right)\right|^{2} d k_{x}^{\prime} d k_{y}^{\prime}}{\int\left|U_{i}\left(k_{x}^{\prime}, k_{y}^{\prime}\right)\right|^{2} d k_{x}^{\prime} d k_{y}^{\prime}} .
$$




\section{2. Динамическая функция пропускания МАОФ: случай почти эКвидистантной сетки частот}

Перейдем к построению динамической функции пропускания МАОФ в условиях, характерных для одной практически значимой задачи в области телекоммуникаций с использованием спектрально-кодируемых сигналов $[4,11,12]$. Имеем оптическое излучение со сплошным спектром в некотором диапазоне длин волн излучения (в данном случае спектральный диапазон может быть относительно небольшим). Необходимо сформировать из этого излучения ряд спектральных каналов с заданным уровнем взаимных межканальных помех при помощи дискретного набора звуковых частот. Заметим, что в рассматриваемых системах связи в целях повышения их спектральной эффективности требуется, чтобы ширина полосы пропускания в отдельном канале была минимальна, а сами каналы расположены эквидистантно и максимально плотно.

Аналогично одночастотному случаю решить эту задачу возможно, зная отклик МАОФ в случае падения монохроматического светового пучка. Наиболее удобно рассмотрение проводить в системе с эквивалентным звуковым пучком, как на рис. 1,c. При этом будем в одночастотном случае использовать выражения (6), (7). Эти выражения содержат безразмерные параметры, которые практически не меняются при преобразовании акустического пучка указанным образом.

Будем использовать модель, учитывающую указанные особенности формирования многоканальной структуры. Введем допущение, что искажение функции пропускания в пределах отдельного канала обусловлено только двумя соседними каналами. Рассмотрим группу из 3 соседних каналов (присвоим им номера $-1,0,1$ ), образованных дифракцией светового пучка на акустическом столбе с почти эквидистантными циклическими частотами $\Omega-\Delta \Omega_{-1}, \Omega, \Omega+\Delta \Omega_{1}$. Очевидно, что значения $\Delta \Omega_{ \pm 1}$ близки. Из-за отклонения частоты необходимо ввести дополнительные параметры, характеризующие расстройку между центральными волнами световых пучков: $\delta_{ \pm 1}=\frac{\Delta \Omega_{ \pm 1}}{2 v_{s}} l_{z}$, причем длина взаимодействия $l_{z}=l_{i} \cos \beta$. Соответственно следует ввести 3 коэффициента $\mathrm{AO}$ связи $V_{-1}, V_{0}, V_{1}$. Теперь можно считать, что эволюцию функций $T_{t, d}$ описывает система (6), но вместо постоянной комплексной связи $V$ следует подставить выражение

$$
\begin{aligned}
V(Z, t)= & V_{0}+V_{-1} \exp \left(j \Delta \Omega_{-1} t-2 j \delta_{-1} Z\right) \\
& +V_{1} \exp \left(-j \Delta \Omega_{1} t+2 j \delta_{1} Z\right) .
\end{aligned}
$$

Таким образом, акустическое поле является профилированным в пространстве и модулированным во времени по гармоническому закону. Можно решить полученную систему численно, „заморозив“ время (за время прохождения света через акустическое поле само поле изменится очень мало). Такое решение будет справедливо в данный момент.
Однако можно получить приближенное аналитическое решение методом последовательных приближений. Действительно, если известны функции $T_{t, n}$ и $T_{d, n}$ в $n$-м приближении, то в следующем приближении (с номером $n+1)$ они будут определены выражениями

$$
\begin{gathered}
T_{d, n+1}=\int_{0}^{Z} V T_{t, n} \exp (2 j F Z) d Z, \\
T_{t, n+1}=1-\int_{0}^{Z} V^{*} T_{d, n} \exp (-2 j F Z) d Z .
\end{gathered}
$$

В качестве нулевого приближения будем использовать (7), т. е. дифракцию на монохроматическом акустическом сигнале, соответствующем центральному каналу. Это естественное допущение, если мы рассматриваем АО дифракцию в пределах отдельного канала. Последовательное применение процедуры (9) дает аналитическое решение со все увеличивающимся количеством слагаемых.

В первом приближении амплитуды центральных порядков не изменятся. Два дополнительных слагаемых будут отличаться по частоте. Следствием этого будут следующие эффекты. Во-первых, поток мощности проходящего и дифрагированного пучков будет модулирован во времени, так как кроме стационарной составляющей остальные слагаемые будут гармонически меняться с циклическими частотами $\Delta \Omega_{ \pm 1}$ и $\Delta \Omega_{-1}+\Delta \Omega_{1}$. Во-вторых, полученное решение уже не соответствует закону сохранения энергии. В следующих приближениях частотный спектр световых пучков обогащается, в том числе комбинационными частотами из-за интермодуляционных искажений. При этом точность соответствия закону сохранения энергии улучшается.

В настоящей работе процедура (9) использована три раза. Из полученных сложных и громоздких аналитических выражений для $T_{t, d}$ выделены гармонические слагаемые, дающие вклад в функцию пропускания в пределах произвольной группы трех соседних каналов. Из-за приближенности решения не полностью выполняется закон сохранения энергии. Поэтому эффективность дифракции рассчитывалась по формуле, формально устраняющей это отличие:

$$
\begin{gathered}
\eta=\frac{\eta_{d}}{\eta_{t}+\eta_{d}}, \\
\eta_{t, d}=\frac{\int\left|U_{i}\left(k_{x}^{\prime}, k_{y}^{\prime}\right)\right|^{2}\left|T_{t, d}\left(k_{x}^{\prime}, k_{y}^{\prime}, 1\right)\right|^{2} d k_{x}^{\prime} d k_{y}^{\prime}}{\int\left|U_{i}\left(k_{x}^{\prime}, k_{y}^{\prime}\right)\right|^{2} d k_{x}^{\prime} d k_{y}^{\prime}} .
\end{gathered}
$$

\section{3. Моделирование характеристик прототипа МАОФ на базе кристалла парателлурита}

Важнейшим $\mathrm{AO}$ материалом является парателлурит $\left(\mathrm{TeO}_{2}\right)$ - сильноанизотропный кристалл класса 422, 


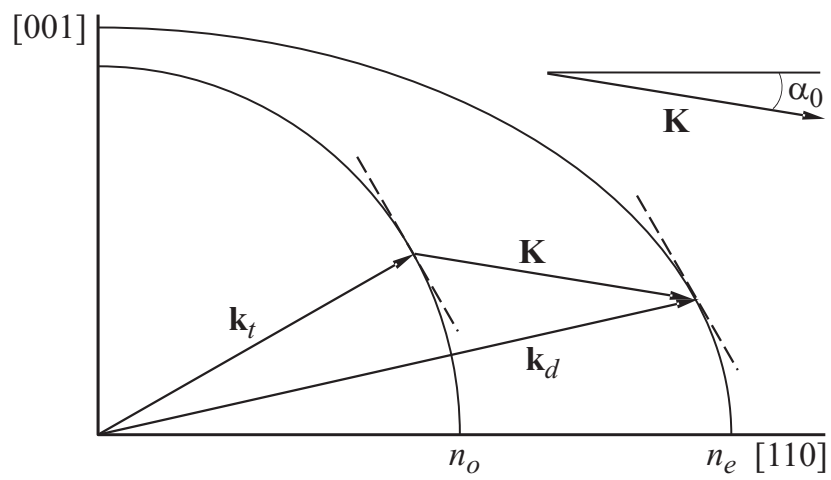

Рис. 2. Векторная диаграмма варианта реализации широкоугольного неколлинеарного фильтра на основе парателлурита.

вследствие чего ярко выражены эффекты сноса и сильного дифракционного искажения звукового пучка [21-23]. В большинстве AO приборов на основе парателлурита используется медленная квазипоперечная акустическая мода вблизи направления [110], для которого скорость звука имеет аномально малое значение $(617 \mathrm{~m} / \mathrm{s})$. Обычно акустическая волна распространяется под некоторым небольшим углом к этому направлению в плоскости (110). Падающий свет направлен под определенным углом к оптической оси (в зависимости от выбранной геометрии) в этой же плоскости. Дифрагированное излучение другой поляризационной моды незначительно отклоняется от падающего излучения.

Для обеспечения большой входной угловой апертуры (например, для обработки изображений) используется так называемая широкоугольная геометрия [24]. В данной геометрии зависимость расстройки $\delta$ от направления падающего света должна быть минимальна. В плосковолновом приближении это соответствует сонаправленному распространению световых пучков в возмущенной звуком области. Таким образом, для этой геометрии характерно совпадение направлений лучевых скоростей взаимодействующих световых волн, что эквивалентно параллельности касательных плоскостей, проведенных через концы векторов $\mathbf{k}_{t, d}$ (рис. 2). В приведенных в разд. 1 обозначениях широкоугольная геометрия соответствует условию $\varphi_{1}=0$.

Как известно, при указанных условиях акустический столб испытывает сильный снос в плоскости дифракции (110), которая является плоскостью АО дифракции $x z$ в нашем анализе. Поэтому световой пучок направлен не вдоль звукового столба, а пересекает его.

Именно широкоугольная геометрия была использована для экспериментов, необходимым условием которых являлось формирование дискретного набора каналов из падающего излучения сплошного спектра [25]. Далее приведены данные моделирования одного из использованных в экспериментах МАОФ. Следует отметить, что моделирование проведено в диапазоне длин волн, намного превышающем ширину полосы излучения источника сплошного спектра.
Рис. 3 иллюстрирует одночастотную функцию пропускания вблизи длины волны, характерной для проведенных экспериментов. Здесь и далее использованы значения АО связи, обеспечивающие эффективность дифракции $80 \%$ в одночастотном случае. Можно отметить, что форма кривой близка к рассчитанной в плосковолновом приближении (это следствие широкоугольной геометрии). Наблюдается также известный систематический сдвиг функции пропускания.

При спектральной перестройке в рассматриваемом диапазоне форма функции пропускания меняется незначительно. Напротив, ширина полосы пропускания $\Delta \lambda$ меняется значительно и нелинейным образом (рис. 4).

Знание этой зависимости позволяет рассчитать плотность размещения каналов. В частности, рис. 5 демонстрирует параметры формируемых каналов, исходя из

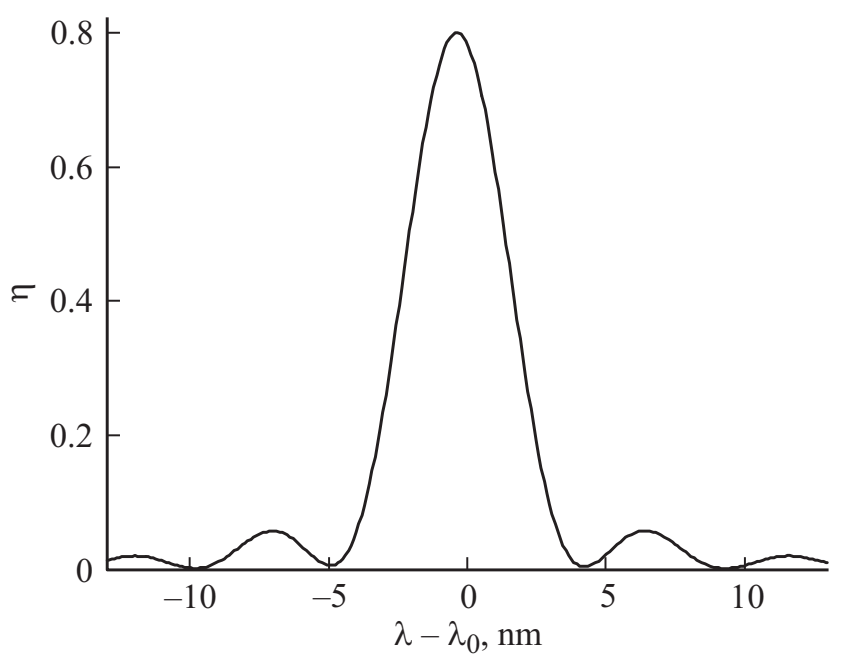

Рис. 3. Одночастотная функция пропускания вблизи выбранного значения длины волны $\lambda_{0}=0.95 \mu \mathrm{m}$.

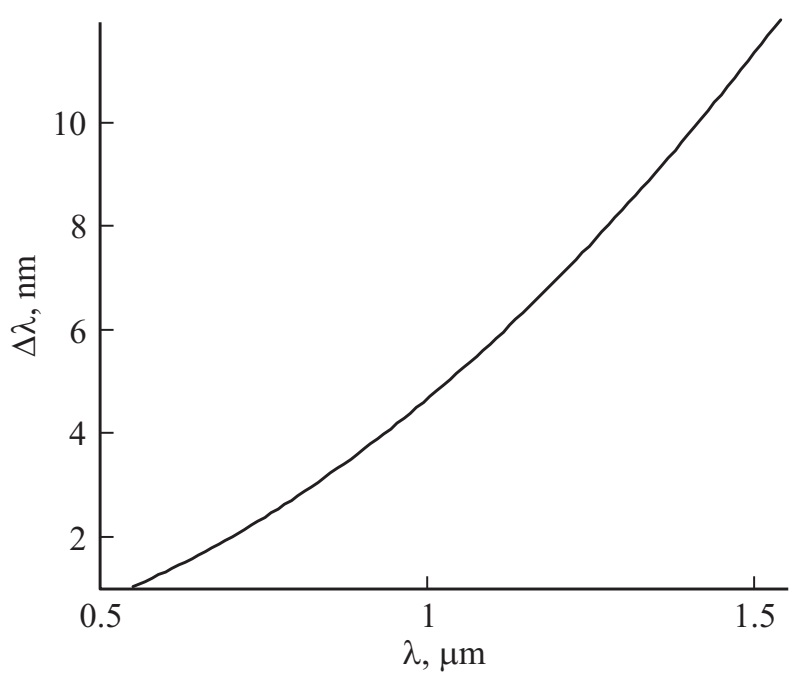

Рис. 4. Зависимость ширины полосы пропускания на половинном уровне (т.е. при эффективности дифракции 40\%) от центральной длины волны фильтруемого излучения. 


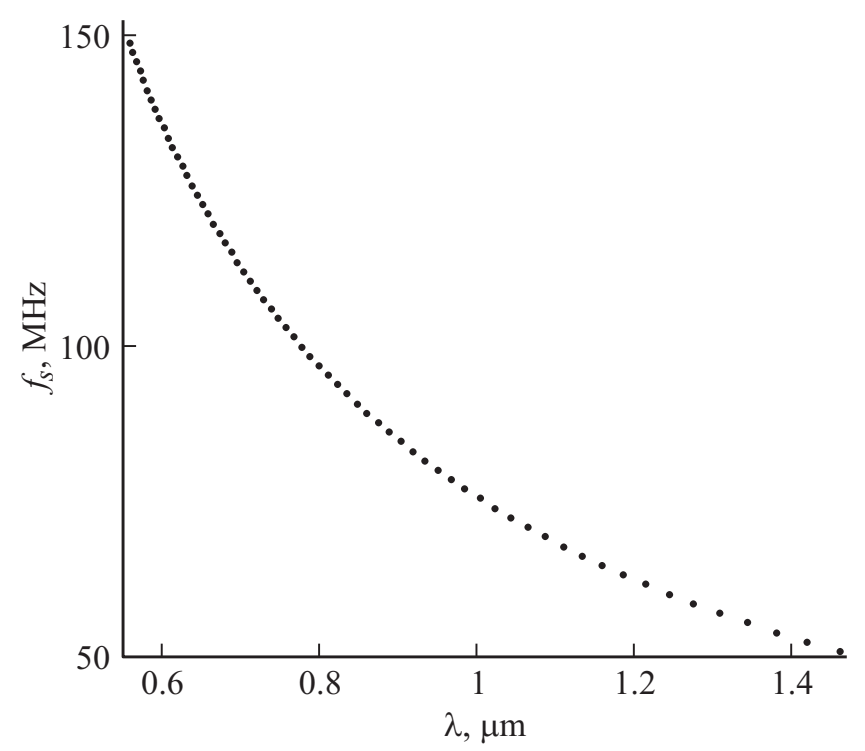

Рис. 5. Совокупность звуковых частот и центральных длин волн, обеспечивающих формирование дискретного набора каналов по выбранному условию.
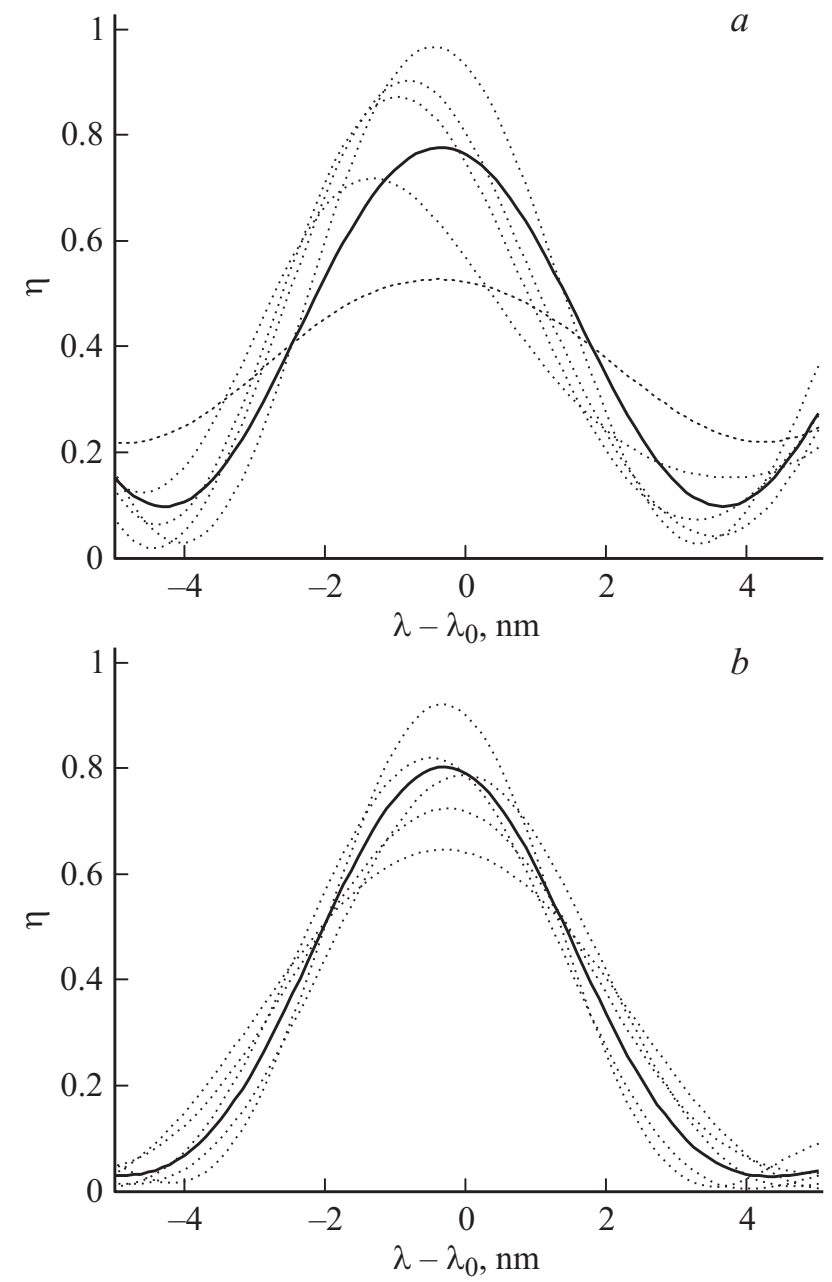

Рис. 6. Мгновенные функции пропускания (штриховые линии) и усредненная функция (сплошная линия) в пределах отдельного канала вблизи длины волны $0.95 \mu \mathrm{m}$. Расстояние между каналами составляет $2(a)$ и $4(b)$ ширины полосы пропускания. того условия, что интервал длин волн между соседними каналами в четыре раза превышает ширину полосы пропускания для данной длины волны.

Рис. 6 демонстрирует несколько мгновенных функций пропускания в пределах отдельного канала (штриховые линии), рассчитанных в случайные моменты времени. Два случая соответствуют различной плотности спектральных каналов. Видно, что для каждой длины волны в пределах канала пропускание меняется в широких пределах даже при условии достаточного разнесения каналов. Как было отмечено, функция пропускания содержит слагаемые, меняющиеся гармонически с разными частотами. В частности, при более плотном расположении каналов наименьшая частота колебаний составляла около $360 \mathrm{~Hz}$, что соответствует циклической частоте $\Delta \Omega_{1}-\Delta \Omega_{-1}$. Если регистрировать поток мощности с определенным усреднением в течение интервала времени, превышающего наибольший период, функция пропускания выглядит стационарной (сплошные линии).

Анализ показывает, что усредненная функция пропускания имеет важные отличия от стационарной картины в одночастотном случае. Во-первых, ни при каком уровне звуковой мощности невозможно достичь наибольшего пропускания для центральной длины волны, рассчитанного в одночастотном случае. Этот уровень в последнем случае достигается вблизи определенного значения АО связи и снижается как при его увеличении, так и при уменьшении. Однако при частичном перекрытии соседних каналов уровень АО связи модулирован во времени, поэтому добиться максимального пропускания не удастся. Таким образом, использование многочастотного режима ограничивает максимальное пропускание в усредненной картине.

Во-вторых, схожие процессы сглаживают пропускание в межканальном пространстве. Действительно, положение минимумов, а также положение и величина боковых лепестков одночастотной функции пропускания определяются уровнем АО связи и расстройкой синхронизма относительно центральной волны. Модуляция АО связи вследствие перекрытия каналов и интермодуляционных эффектов приводит к быстрой перестройке положения минимумов и побочных максимумов. В результате усреднения исчезают ярко выделенные минимумы и боковые максимумы, форма межканального пропускания становится более гладкой, минимальное пропускание увеличивается. Эти особенности хорошо видны в обоих случаях на рис. 6.

\section{Заключение}

1. Построена теория АО фильтрации светового пучка в поле монохроматического акустического столба. Получены аналитические выражения для пространственных спектров световых пучков и функции пропускания с учетом расхождения пучков и их дифракционного расплывания. 
2. Предложен метод, позволяющий приближенно рассчитать динамическую функцию пропускания многочастотного АО фильтра в том случае, когда функции пропускания, соответствующие отдельным акустическим составляющим, расположены не ближе, чем несколько ширин полос пропускания. Метод основан на последовательных приближениях и дает аналитическое решение. Показано, что динамическая картина пропускания кроме стационарной составляющей содержит большое количество гармонически меняющихся компонент.

3. Проведен анализ формирования почти эквидистантной многоканальной спектральной картины при фильтрации излучения широкого пространственного спектра. Обнаружено, что динамическая функция пропускания вследствие гармонических составляющих меняется в определенных пределах для каждой длины волны, попадающей в выбранный спектральный канал. При усреднении потока мощности дифрагированного света в течение времени, большей определенной величины, формируется усредненная функция пропускания. Она обнаруживает новые черты, не свойственные одночастотной функции пропускания. В частности, уменьшается максимально достижимая эффективность дифракции для отдельного канала, межканальное пропускание становится более гладким по форме, увеличивается минимальное пропускание.

4. Выполнен вычислительный эксперимент, моделирующий формирование почти эквидистантной многоканальной спектральной картины при фильтрации излучения широкого пространственного спектра. Для моделирования выбран АО широкоугольный неколлинеарный фильтр на основе кристалла парателлурита, использованный ранее в экспериментах по оптическому кодированию.

Работа выполнена при финансовой поддержке Российского фонда фундаментальных исследований (проект № 16-02-00124-a).

\section{Список литературы}

[1] Hinkov I., Hinkov V., Iversen K., Ziemann O. // Electron. Lett. 1995. V. 31. N 5. P. 384.

[2] Goto N., Miyazaki Y. // Trans. IEICE. 1987. V. E70. N 10. P. 981.

[3] Проклов В.В., Бышевский-Конопко О.А., Филатов А.Л. // Радиотехника. 2000. № 1. С. 50.

[4] Проклов В.В., Бышевский-Конопко О.А., Григорьевский В.И. // Радиотехн. и электрон. 2013. Т. 58. № 9. С. 905; Proklov V.V., Byshevskii-Konopko O.A., Grigor'evskii V.I. // J. Communications Technology and Electronics. 2013. V. 58. N 9. P. 891-900. doi 10.1134/S1064226913090118

[5] Proklov V.V., Byshevski-Konopko O.A., Lugovskoi A.V. // Acta Physica Polonica A. 2015. V. 127. P. 29-35. doi 10.12693/APhysPolA.127.29

[6] Hecht D.L. MultifrequencyAcoustooptic Diffraction // IEEE Trans. on Sonics and Ultrasonics. 1977. V. SU-24. N 1. P. 7-18.
[7] Proklov V.V., Antonov S.N., Rezvov Yu.G., Vainer A.V. // Proc. IEEE Int. Ultrasonics Symposium. 2006. P. 248. doi 10.1109/ULTSYM.2006.75

[8] Antonov S.N., Vainer A.V., Proklov V.V, Rezvov Yu.G. // Appl. Optics. 2009. V. 48. N 7. P. 171. doi 10.1364/AO.48.00C171

[9] Shnitser P., Agurok I., Sandomirsky S. et.al. // Proc. SPIE. 2000. V. 4025. P. 30.

[10] Molchanov V.Ya., Chizhikov S.I, O.Yu. Makarov et. al. // Appl. Optics. 2009. V. 48. P. 118.

[11] Proklov V.V., Byshevski-Konopko O.A., Filatov1 A.L., Lugovskoi A.V., Pisarevsky Yu.V. // J. Physics: Conference Series. 2016. V. 737. P. 012060. doi 10.1088/1742-6596/737/1/0120602

[12] Byshevski-Konopko O.A., Proklov V.V., Filatov A.L., Lugovskoi A.V., Korablev E.M. // Physics Procedia. 2015. V. 73. P. 251-256. doi 10.1016/j.phpro.2015.09.166

[13] Бышевский-Конопко О.А., Проклов В.В., Луговской А.В., Кораблев E.M. // Материалы VI Международной конференции по фотонике и информационной оптике. М.: НИЯУ МИФИ, 2017. С. 20-21.

[14] Магдич Л.Н., Молчанов В.Я. Акустооптические устройства и их применение. М.: Сов. Радио, 1978.

[15] Korpel A. Acousto-optics, Marcel Deccer, 1988; Kopneл A. Акустооптика. М.: Мир, 1993.

[16] Балакший В.И., Парыгин В.Н., Чирков Л.Е. Физические основы акустооптики. М.: Радио и связь, 1985.

[17] Задорин А.C. Динамика акустооптического взаимодействия. Томск, 2004.

[18] Задорин А.С., Шандаров С.М., Шарангович С.Н. Акустические и акустооптические свойства монокристаллов. Томск, 1987.

[19] Балакший В.И., Волошин А.С. // Опт. и спектр. 2011. T. 110. № 5. C. 840; Balakshy V.I., Voloshin A.S. // Opt. Spectroscopy. 2011. V. 110. N 5. C. 788-794. doi $10.1134 / \mathrm{S} 0030400 \mathrm{X} 11050031$

[20] Балакший В.И., Волошин А.С., Молчанов В.Я. // Опт. и спектр. 2014. Т. 117. № 5. С. 827; Balakshy V.I., Voloshin A.S., Molchanov V.Y. // Opt. Spectrosc. 2014. V. 117. N 5. C. 801-806. doi 10.1134/S0030400X14110046

[21] Балакший В.И., Манщевич С.Н. // Акустический журнал. 2012. T. 58. № 5. C. 600; Balakshy V.I., Mantsevich S.N. // Acoustical Physics. 2012. V. 58. N 5. P. 549-557. doi 10.1134/S1063771012050041

[22] Антонов С.Н., Вайнер А.В., Проклов В.В., Резвов Ю.Г. // ЖТФ. 2010. Т. 80. № 3. C. 97; Antonov S.N., Vainer A.V., Proklov V.V., Rezvov Y.G. // Technical Physics. The Russian J. Appl. Phys. 2010. V. 55. N 3. P. 413-422. doi 10.1134/S1063784210030138

[23] Антонов С.Н., Вайнер А.В., Проклов В.В., Резвов Ю.Г. // ЖТФ. 2013. Т. 83. № 12. C. 11; Antonov S.N., Vainer A.V., Proklov V.V., Rezvov Y.G. // Technical Physics. The Russian J. Appl. Phys. 2013. V. 58. N 12. P. 1715-1720. doi 10.1134/S1063784213120037

[24] Chang I.C. // Appl. Phys. Lett. 1974. V. 25. N 7. P. 370-372.

[25] Proklov V.V., Byshevski-Konopko O.A., Filatov A.L., Lugovskoi A.V., Pisarevsky Yu.V. // J. Phys.: Conf. Ser. 2016. V. 737. N 1. P. 1-6. 